\title{
Computationally optimized formulation for the simulation of composite materials and delamination failures
}

\author{
Xavier Martinez ${ }^{\mathrm{a}, \mathrm{b}, *}$, Fernando Rastellini ${ }^{\mathrm{a}, \mathrm{c}}$, Sergio Oller $^{\mathrm{a}, \mathrm{d}}$, Fernando Flores ${ }^{\mathrm{a}, \mathrm{e}}$, Eugenio Oñate ${ }^{\mathrm{a}, \mathrm{d}}$ \\ a International Center for Numerical Methods in Engineering (CIMNE), Gran Capità s/n, Edifici C1, Campus Nord., Barcelona 08034, Spain \\ ${ }^{\mathrm{b}}$ Departament de Ciència i Enginyeria Nàutica, UPC, Pla de Palau 18, Barcelona 08003, Spain \\ ${ }^{\mathrm{c}}$ Quantech ATZ S.A. Gran Capità 24. Edifici Nexus, Barcelona 08034, Spain \\ ${ }^{\mathrm{d}}$ Departament de Resistència de Materials i Estructures a l'Enginyeria, UPC. Gran Capità s/n, Edifici C1, Campus Nord, Barcelona 08034, Spain \\ ${ }^{\mathrm{e}}$ Departamento de Estructuras, Universidad Nacional de Córdoba, Casilla de Correo 916, Cordoba 5000, Argentina
}

\section{A R T I C L E I N F O}

\section{Article history:}

Received 27 June 2010

Received in revised form 20 September

2010

Accepted 21 September 2010

Available online 1 October 2010

\section{Keywords:}

A. Laminates

B. Delamination

C. Damage mechanics

C. Finite element analysis

\begin{abstract}
A B S T R A C T
The numerical simulation of complex failure modes of composite materials, such as delamination, can be computationally very demanding, as it requires special elements and/or numerical strategies to characterize damage onset and propagation. This work presents several formulations developed to optimize the computational performance of an explicit finite element code designed specifically for the simulation of large scale composite structures. The composite mechanical performance is obtained with the matrixreinforced mixing theory, a simplified version of the serial/parallel mixing theory that does not require an iterative procedure or the calculation of the tangent stiffness matrix. The number of elements required to perform the simulation is reduced by stacking several layers inside a single finite element. This work also proposes a modification of the isotropic damage law, capable of taking into account the residual strength provided by friction in type II fracture modes. The ability of these formulations to successfully predict the mechanical performance of composite materials is assessed with the ply drop-off test. In this test a laminate with a change of thickness in its mid-span is loaded until it breaks due to a delamination process. The formulation proposed obtains a very accurate prediction of the experimental response of the test, as it provides a very good characterization of the initial laminate stiffness, the delamination onset, and its propagation along the specimen.
\end{abstract}

(ㄷ) 2010 Elsevier Ltd. All rights reserved.

\section{Introduction}

The use of new materials in structural applications implies dealing with new failure processes, not existing in traditional materials. One of these is the delamination failure found in laminated composites. This failure is produced by the loss of adherence between the different layers of the composite, which leads to a reduction of the section strength and stiffness. Delamination is a critical failure mode in composites, not because it causes the structure to break, but because it splits the composite in different layers not connected between them, modifying the structural performance of the composite and leading to its failure because of other mechanical phenomenons such as buckling, excessive vibration, or loss of fatigue life [1]. With this scope, Bolotin [2] defines two types of delamination processes: internal and near the surface. While the first ones affect the whole composite performance, the

\footnotetext{
* Corresponding author at: Departament de Ciència i Enginyeria Nàutica, UPC, Pla de Palau 18, Barcelona 08003, Spain. Tel.: +34 934017306.

E-mail address: x.martinez@upc.edu (X. Martinez).
}

second ones only affect the external layers of the composite and lead to a local buckling failure.

Delamination is usually characterized by two different processes: delamination onset and crack propagation. Delamination onset is defined by the capability of the composite to resist the initial apparition of damage. Delamination propagation is driven by the damage tolerance of the material, which can be understood as the capability of a damaged material to sustain a load maintaining its structural performance.

A detailed survey of the historical treatment of the delamination problem, and of the procedures used nowadays to deal with it, can be found in Refs. $[1,3,4]$. These surveys show that all authors that have proposed formulations and simulation methods to characterize the delamination failure agree on solving the problem by representing the two processes that take place: delamination onset, or crack initiation, and its propagation along the composite. Crack initiation can be obtained by several means, such as comparing the strain or stress states of the material in the region where delamination initiates, with a critical one [5-8]; in terms of the traction applied to the specimen, versus its relative displacement [9-11]; or based on the maximum strain energy that can 
be developed by the element, using the energy release rate (ERR) $[12,13]$. Delamination propagation is usually treated opening the mesh to simulate the crack. Among the different procedures developed to open the mesh, it is worthy to mention the virtual crack closure technique (VCCT) [14]. This is based on the assumption that when a crack is extended, the energy required to open the crack is the same required to close it. Another procedure that has increased its popularity recently consists in using elements with a cohesive zone model [6]. The cohesion elements are placed in the interface of the layers that can be affected by delamination, and the fracture propagation in these elements is obtained using damage mechanics.

Despite conceptual differences between formulations for the simulation of delamination, such as the VCCT [14] or the cohesive method [6-9], all available methodologies for delamination rely on pre-defined interfaces that constrain the path of the crack. If the path of the delamination is unknown, cohesive or fracture surfaces must be placed between all plies, which is computationally expensive

In contrast to the scope used in known literature to solve the delamination problem, this work uses continuum mechanics to simulate the delamination initiation and propagation, without making any distinction of the elements in which delamination will take place. The proposed procedure is based on simulating the composite with a simplified version of the serial/parallel mixing theory [15], specially developed to be implemented in an explicit finite element code. The proposed procedure also requires simulat

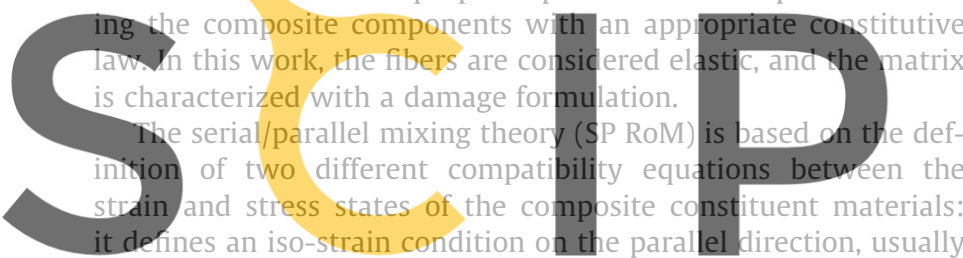
the fiber direction, and it defines an iso-stress condition on the serial direction, usually the remaining directions. Using these

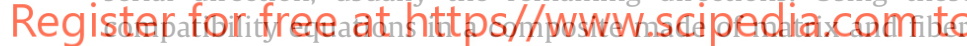
if the matrix structural capacity is lost due to excessive shear stresses, the iso-stress condition also reduces the shear capacity of fiber, and consequently the composite serial strength is also reduced. It has been already proved in [16] that the structural failure just described is equivalent to a delamination failure. This work presents a new version of the SP RoM, called the matrixreinforced mixing theory (MR RoM). This formulation has been developed with the aim of reducing the computational cost of the SP RoM, without affecting significantly its simulation performance, in particular, its ability to predict delamination processes. The new formulation is validated with the ply drop-off test by comparing experimental values with the values obtained from a numerical simulation.

The following section describes the new mixing theory developed, the MR RoM, and how it is implemented in a finite element code. It also describes the method used to simulate laminates with solid elements: by stacking several layers into a single finite element. This section also presents a new damage formulation, capable of taking into account the residual strength of a delaminated composite due to the friction between the delaminated surfaces. Next section describes the test simulated. The results obtained from the simulation are studied and compared to the experimental ones. Finally, last section presents the conclusions drawn from the simulation performed.

\section{Formulation}

The simulations included in this work are made with Compack, a finite element package result of the collaboration between
CIMNE and QUANTECH. The mechanical performance of composites is obtained with the serial/parallel mixing theory [15]. The code contains implicit and explicit solution strategies. While it has already been proved the ability of the implicit solver to simulate a delamination process [16], this work will show how the explicit solver deals with it. In the following are described the formulations developed and the procedures used for that purpose.

\subsection{Characterization of composite materials}

\subsubsection{Serial/parallel mixing theory}

The serial/parallel mixing theory (SP RoM) is based on the differentiation and separation of the composite mechanical response into two components: a parallel and a serial one. The parallel component corresponds to the direction in which fibers are aligned, and the serial component corresponds to the rest of directions. The separation of the composite response in these two directions has been already used by many authors $[17,18]$ to obtain the elastic components of fiber reinforced composites (FRP); however, none of these authors provide a formulation capable of predicting the composite performance when one of its constituents gets into the non-linear range (due to damage or plasticity, for example).

The SP RoM acts as a constitutive equation manager, and is capable of successfully predict the structural performance of the composite, taking into account the specific behavior of the composite in its parallel and serial direction, as well as the non-linearities of the composite components [15,19]. To do so, the strain

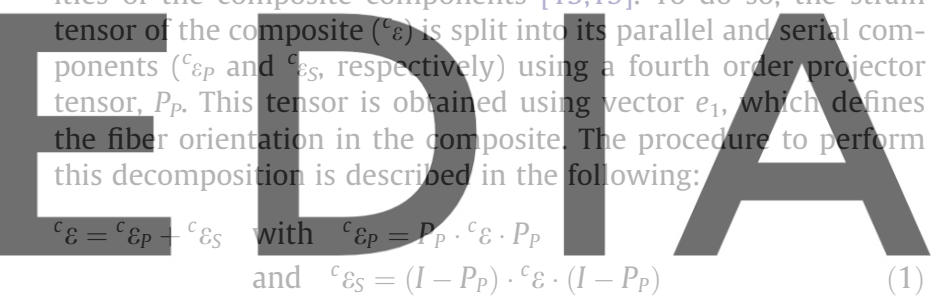

dowipibade the version witho idtethe watermark Once knowing the parallel and serial components of the composite, it is possible to apply to each one the compatibility conditions that define how the composite constituents, fiber and matrix, interact between them:

Parallel direction : $\left\{\begin{array}{l}{ }^{c} \varepsilon_{P}={ }^{f} \varepsilon_{P}={ }^{m} \varepsilon_{P} \\ { }^{c} \sigma_{P}={ }^{f} k \cdot{ }^{f} \sigma_{P}+{ }^{m} k \cdot{ }^{m} \sigma_{P}\end{array}\right.$

Serial direction : $\left\{\begin{array}{l}{ }^{c} \varepsilon_{S}={ }^{f} k \cdot{ }^{f} \varepsilon_{S}+{ }^{m} k \cdot{ }^{m} \varepsilon_{S} \\ { }^{c} \sigma_{S}={ }^{f} \sigma_{S}={ }^{m} \sigma_{S}\end{array}\right.$

The strains of fiber and matrix are obtained from the composite strains, using the compatibility Eqs. (2) and (3). Once the strains are known, the stresses of both components are calculated according to their own constitutive law. It is important to remark that each composite constituent can be modeled using an independent constitutive equation; and that the SP RoM allows using any material law such plasticity, damage, or visco-elasticity. The resultant stresses, obtained from the constitutive equation of fiber and matrix, have to fulfill the compatibility Eqs. (2) and (3).

Compatibility equations are easily matched in the parallel direction, as the parallel strains of fiber and matrix are obtained straightforward from the composite strains. However, the compatibility equations are not fulfilled so easily in the serial direction: it is necessary an initial prediction of either fiber or matrix serial strains, in order to obtain the other one. And the serial strains considered must be chosen wisely, so that the serial stresses obtained for both components end up being the same. If fiber and matrix have a linear elastic behavior, it is possible to calculate the serial 
strains that fulfill the compatibility equation using Hooke's law. However, if any of the two component materials is non-linear, it is necessary to use an iterative procedure in order to calculate the serial strains that fulfill Eq. (3). This procedure, based on a Newton-Raphson scheme, is fully described in [15]. The ability of the SP RoM to successfully predict the mechanical performance of composite materials, once their components have reached the non-linear range, has been proved in previous works such as $[15,19,20]$.

\subsubsection{Matrix-reinforced mixing theory}

An explicit time integration method requires evaluating many times the constitutive equation of the material, as the time step in which the problem is divided is very small in order to avoid numerical instabilities. Under these conditions, a constitutive law like the SP RoM, that requires an iterative procedure to obtain the composite stress, makes the calculation very time consuming, being impossible to apply the formulation to perform large simulations of composite structures. So, it becomes imperative to develop a simplified version of the serial/parallel mixing theory, computationally less demanding.

This work proposes what will be called the Matrix-reinforced mixing theory (MR RoM), which is based on the concept of vanishing fiber diameter defined by Dvorak and Bahei-el-Din [21]. The MR RoM states that in multi-layer laminates the contribution of fibers to the stiffness and strength in the transverse direction of a single layer can be neglected, especially if the laminate has several

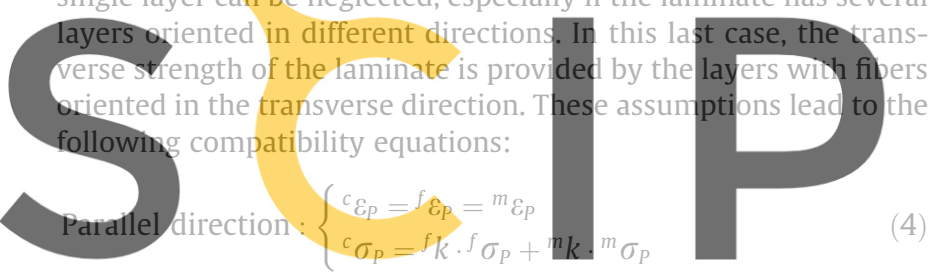

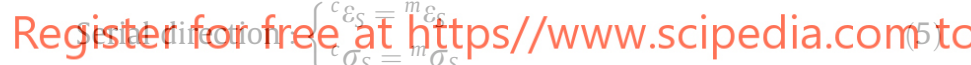

With these new compatibility equations it is possible to calculate directly the parallel components of the fiber and matrix strains, as well as their serial components. So it is possible to evaluate the constitutive equations of both materials, thus avoiding the iterative procedure required by the SP RoM. Once the stresses of fiber and matrix are known, the composite stresses are calculated with the compatibility Eqs. (4) and (5).

Another advantage of the MR RoM is that it does not require the evaluation of the tangent stiffness tensor of the composite constituents, which is required by the iterative process of the SP RoM. And, since this tensor is not needed by the explicit integration scheme either, its calculation is not required at any time. This is another important reduction of the computational cost in the simulation, as this tensor is not always easy to obtain and, in some cases, requires of very time consuming procedures such as a numerical derivation [19].

Therefore, taking into account that the iterative procedure of the SP RoM will require performing a minimum of twice the number of operations required in a non-iterative process, and that the number of operations that have to be performed to calculate tangent stiffness matrix is larger than the number of operations performed by the MR RoM; it can be said that in a non-linear case, the MR RoM reduces by a factor of three or more the computational cost of calculating the composite stresses, when compared with the SP RoM.

In order to prove the capability of the MR RoM to simulate laminated composites, in the following is compared the stiffness obtained for a laminate with the SP RoM and the MR RoM. Two different numerical simulations have been performed with each formulation, applying in the first one a longitudinal load and in the second one a transverse load. The composite simulated is a $25 \times 25 \mathrm{~mm}$ laminate specimen, manufactured with 18 layers of IMS/977.2 prepeg with the following stacking sequence $\left(-45 / 0_{2} /\right.$ $\left.+45 /+90 /+45 / 0_{2} /-45\right)_{s}$. The mechanical properties of this composite, and of its constituent materials, are described in Section 3.1, when describing the experimental test performed to validate the formulation presented. The longitudinal load corresponds to the direction of the layers with fibers oriented at $0^{\circ}$. The results obtained for each simulation are shown in Table 1, which shows the final load and displacement obtained for each simulation. These two values have been used to calculate the laminate stiffness in each direction. The comparison of the longitudinal and transverse stiffness shows that both formulations provide nearly the same results, with a discrepancy slightly larger (but smaller than a $1 \%$ ) on the transverse stiffness than in the longitudinal one.

The differences shown in Table 1 , regarding the transverse stiffness of the composite, are larger if the transverse displacement studied corresponds to the deformation of the composite due to Poisson effects. These results have been obtained from the longitudinal load simulation and are shown in Table 2. This table shows that the difference in the transverse stiffness obtained when comparing the SP RoM and the MR RoM results is nearly a 19\%; this difference is reduced to a $15 \%$ when comparing the "through the thickness" stiffness. Despite the differences shown by this table, it has to be noted that the contribution of the Poisson effect to

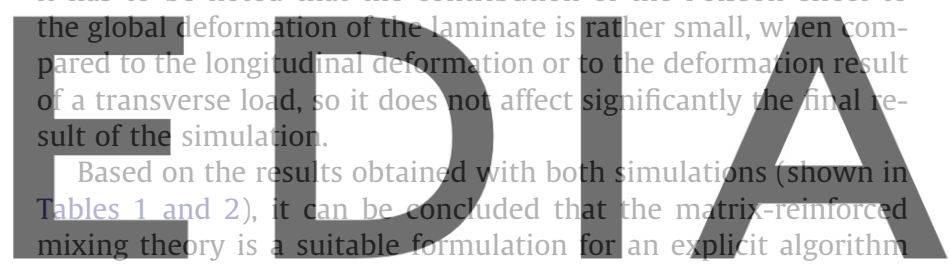

because, although it is less precise than the serial/parallel mixing theory in the transverse direction, it has the advantage of being sig-

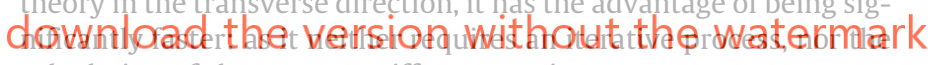
calculation of the tangent stiffness matrix.

\subsubsection{Simulation of laminates using solid elements}

The best way to simulate laminate composites using solid elements is by placing an element in each layer of the laminate. However this is not feasible in most cases as the number of layers can be in the order of hundreds. Therefore, it is necessary to use a procedure capable of compacting some layers in a single element, as it is shown in Fig. 1, in which is represented a three-dimensional solid finite element containing three layers of a laminate oriented at $(0 / 90 / 0)$.

Because a solid finite element does not have bending efforts in its formulation, in-plane strains $\left(\varepsilon_{1}\right.$ and $\left.\varepsilon_{2}\right)$ must be equal for all layers contained in laminate depicted in Fig. 1; therefore, these layers present a parallel behavior and the mixing theory can be applied to them. On the other hand, in the out-of-plane direction $\left(\varepsilon_{3}\right)$, layers present a serial distribution and the performance of

Table 1

Results obtained for a laminate with the SP RoM and the MR RoM.

\begin{tabular}{llllll}
\hline & \multicolumn{2}{l}{ Longitudinal load } & & \multicolumn{2}{l}{ Transverse load } \\
\cline { 2 - 3 } \cline { 5 - 6 } & SP RoM & MR RoM & & SP RoM & MR RoM \\
\hline Load $(\mathrm{kN})$ & 171.92 & 171.84 & & 19.69 & 19.56 \\
$\begin{array}{l}\text { Displacement } \\
\quad(\mathrm{mm})\end{array}$ & 0.20 & 0.20 & & 0.10 & 0.10 \\
$\begin{array}{l}\text { Stiffness }(\mathrm{kN} / \mathrm{m}) \\
\text { Error }\end{array}$ & $8.596 \times 10^{5}$ & $8.593 \times 10^{5}$ & & $1.969 \times 10^{5}$ & $1.956 \times 10^{5}$ \\
& & $0.04 \%$ & & $0.64 \%$ \\
\hline
\end{tabular}


Table 2

Transverse stiffness due to Poisson obtained for a laminate. SP RoM versus MR RoM.

\begin{tabular}{|c|c|c|c|c|}
\hline & \multicolumn{2}{|c|}{ Transverse direction } & \multicolumn{2}{|c|}{ Through the thickness direction } \\
\hline & SP RoM & MR RoM & SP RoM & MR RoM \\
\hline Longit. load (kN) & 171.92 & 171.84 & 171.92 & 171.84 \\
\hline Transv. displ. (mm) & 0.072 & 0.088 & 0.007 & 0.006 \\
\hline Stiffness $(\mathrm{kN} / \mathrm{m})$ & $2.401 \mathrm{E}+06$ & $1.947 \mathrm{E}+06$ & $2.426 \mathrm{E}+07$ & $2.796 \mathrm{E}+07$ \\
\hline Error & & $18.9 \%$ & & $-15.3 \%$ \\
\hline
\end{tabular}

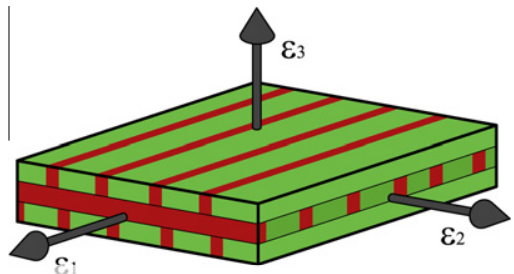

Fig. 1. Strains in a 3D finite element representing a laminate.

the composite should be obtained using an inverse mixing theory. However, in a laminate structure, out-of-plane strains are very small compared with the in-plane strains, so the error obtained in the simulation, if all layers are defined with the same strain value in the through the thickness direction, will be negligible. Defining an iso-strain behavior for all layers existing in the fi

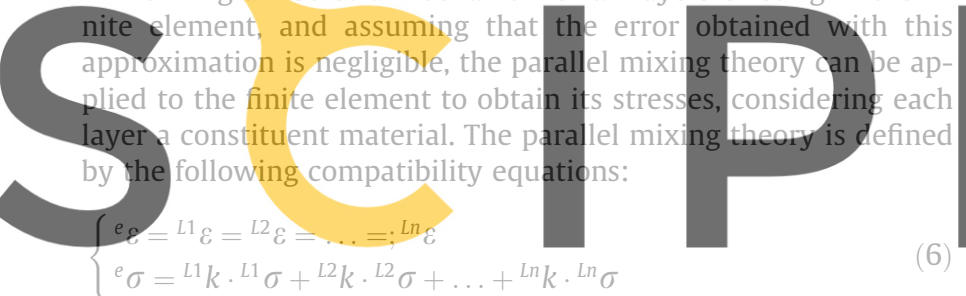

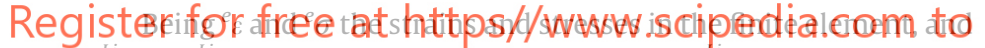
${ }^{L i}{ }_{\varepsilon}$ and ${ }^{L i} \sigma$ the strains and stresses of layer $i ;{ }^{L i} K$ is the volumetric participation of layer $i$ in the finite element. Once the strain in each layer is know, the stresses will be computed using the matrix-reinforced mixing theory, in order to take into account the unidirectional behavior of fiber material in the layer.

With the procedure described, it has to be noted that to consider the bending effects in laminate composites, it is necessary to simulate the laminate with more than one finite element along its thickness. However, this has to be done in all simulations in which laminate structures are simulated with solid elements, either if the material is a laminated composite or if it is a single homogeneous material. The advantage of the formulation described is that laminates with large number of layers, like the ones used in aeronautical applications, can be simulated with solid elements representing stacks of several layers, reducing significantly the computational time required to perform the simulation, as this time is proportional to the mesh size.

\subsection{Characterization of matrix material: damage formulation}

\subsubsection{Isotropic damage formulation}

Material degradation in a continuum solid due to a fracture process can be simulated with a damage formulation. This formulation takes into account the reduction of the effective area of the material, reducing its stiffness properties. The procedure proposed in this work to simulate delamination uses a damage constitutive law to predict the matrix mechanical performance. The damage model considered is the isotropic continuum damage formulation developed by Oliver et al. [22,23].
An isotropic damage formulation is based in the introduction of a scalar internal variable, the damage parameter $d$, that represents the level of degradation of the material. This variable takes values ranged between 0 and 1 , being zero when the material is not damaged and one when the material is completely damaged. The damage parameter is used to transform the real damaged stress tensor, $\sigma$, into an effective stress tensor, $\sigma_{0}$. The effective stress is the stress that will be obtained if the material is not damaged. Therefore, the relation between the damaged stress and the strain in the material depends on the damage parameter and the elastic stiffness tensor $\left(C_{0}\right)$ :

\section{$\sigma \equiv(1-d) \cdot \sigma_{0}=(1-d) \cdot C_{0}: \varepsilon$}

When a damage formulation is used to describe the constitutive performance of a given material, it is necessary to define: (1) The stress value at which damage starts and (2) the evolution of the damage parameter as the load applied increases. Both aspects are

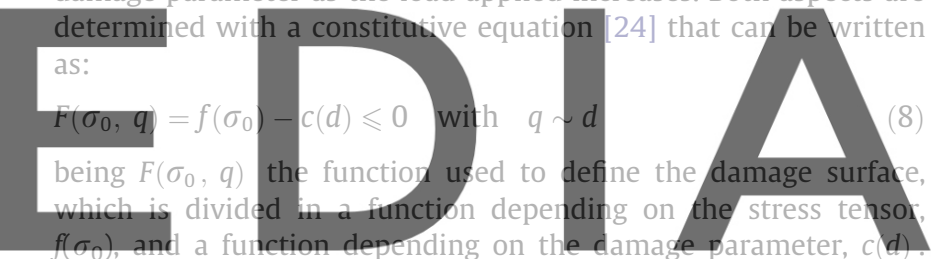

Damage starts the first time that the value of $f\left(\sigma_{0}\right)$ is equal or larger than $f(d)$

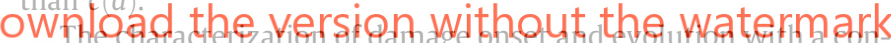
stitutive equation makes possible to use any model already defined in literature, such as Von-Mises, Mohr-Coulomb or Drucker-Prager, to characterize the mechanical behavior of the damaged material. Of all of them, the present work uses the norm of the principal stresses, with a different degradation path for tension and compression loads. This can be written as:

$f\left(\sigma_{0}\right)=\rho \cdot\left\|\sigma_{I}\right\|$

with $\sigma_{I}$ the principal stress tensor and $\rho$ a function that weights the proportion of tension and compression stresses that are applied to the material. This weight function is defined as:

$\rho=r_{0} N+\left(1-r_{0}\right) \quad$ with $N=\tau_{c} / \tau_{t}$ and $\quad r_{0}=\frac{\sum_{I=1}^{3}\left\langle\sigma_{I}\right\rangle}{\sum_{I=1}^{3}\left|\sigma_{I}\right|}$

being $\tau_{c}$ and $\tau_{t}$ the ultimate strength of the material in compression and tension, respectively, and $\langle x\rangle$ the McAully function, which is defined as: $\langle x\rangle=0.5 \cdot(x+|x|)$

The variation of the damage parameter is obtained using the damage consistency parameter and the Kuhn-Tucker condition [23], being possible to explicitly integrate the damage internal variable to obtain:

$c(d)=\max \left\{\tau_{c}, \max \left\{f\left(\sigma_{0}\right)\right\}\right\}$

$d=G\left(f\left(\sigma_{0}\right)\right)$

Being G a C1 positive function, with its derivative also positive. The function $\mathrm{G}$ defines the softening evolution of the material. The 
present work uses an exponential softening, which is defined with the following expression:

$G\left(f\left(\sigma_{0}\right)\right)=1-\frac{\tau_{c}}{f\left(\sigma_{0}\right)} e^{A\left(1-\frac{f\left(\sigma_{0}\right)}{\tau_{c}}\right)}$

where $A$ is a parameter that depends of the fracture energy of the material. For an exponential softening this parameter can be calculated as:

$A=\frac{1}{\frac{g_{c} C_{0}}{\tau_{c}^{2}}-\frac{1}{2}} \quad$ with $\quad g_{c}=\frac{G_{c}}{l_{f}}$

Being $C_{0}$ the elastic stiffness of the material, $G_{c}$ the fracture compression energy of the material and $l_{f}$ the fracture length. This last parameter corresponds to the length of the finite element in which is applied the damage formulation. This parameter is required to take into account that the energy dissipated in a fracture process depends on the element size. The introduction of the fracture length in the formulation makes the degradation process mesh independent [16].

\subsubsection{Isotropic damage with friction}

As has been described, the damage parameter $d$ provides the degree of deterioration of the material. When $d=1$ the material is considered to be fully deteriorated, and it cannot provide further strength to the structure: stresses in the material are null. In a finite element simulation, this situation implies that the elements found around the damaged element can move freeiy, without any structural restriction. This mechan the fractured surface is perpendicu to the structure, which corresponds However if the fracture is in mode II o statement is not fully correct, as the fracture surface can still develop some strength through effects such a fi

Despite all existing research dealing with the delamination problem, the effect of friction on delamination is seldom consid-

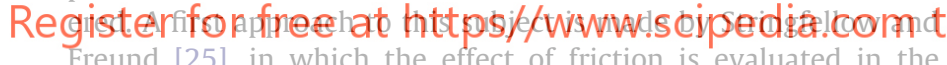
delamination of a thin from a substrate. In their approach, the authors develop an anaiytical modei that is based on the disiocations found in crystal materials, which are represented by the Burger's vector [26]. The model is implemented in a finite element code and is used to prove that friction is one of the main responsible effects determining the toughness strength of mode II fractures.

Regarding the effect of friction in delamination of composite laminates, Davidson and Sun [27] have studied the role of friction, among other parameters such as geometry and fixture compliance, in the delamination toughness obtained in mode II delamination tests. These authors add a new term to the energy balance used to obtain the energy release rate predicted by the mode II delamination tests,

$G=\frac{1}{B}\left[\frac{\partial\left(W_{e}-U\right)}{\partial a}-\frac{\partial W_{f}}{\partial a}\right]$

with $W_{e}$ the work done by the external forces, $U$ the strain energy, $W_{f}$ the work of the frictional forces, $B$ the sample width, and $a$ the fracture length. The variation of the work done by frictional forces is obtained as the product of the tangential force by the tangential displacement. The authors include the effect of friction, among others, in the finite element simulation of three- and four-node bending tests in order to determine the reliability of the results obtained with each one of these tests.

The objective of all the studies found in literature, like the ones just presented, is to assess the effects of friction in delamination toughness. However, no study has been found in which frictional effects are considered to take into account the residual strength provided by the fractured surface in mode II delamination. Hereafter is proposed a first approach to take into account this phenomenon, based on the addition of an empirical parameter into the isotropic damage formulation.

In order to take into account the residual strength provided by the fractured surface in a mode II fracture process, such as delamination, a friction damage parameter, $d^{\text {fric }}$, is introduced into the damage formulation. This parameter defines the residual stress that remains in the material due to friction effects. The definition of this residual stress is equivalent to define a maximum value that can be reached by the damage parameter $d$. Therefore, the relation between the real damaged stress tensor $\sigma$ and the effective stress tensor $\sigma_{0}$ becomes:

$\sigma=\left(1-d^{*}\right) \cdot \sigma_{0} \quad$ with $\quad d^{*}=\min \left\{d, d^{\text {fric }}\right\}$

The frictional damage parameter,
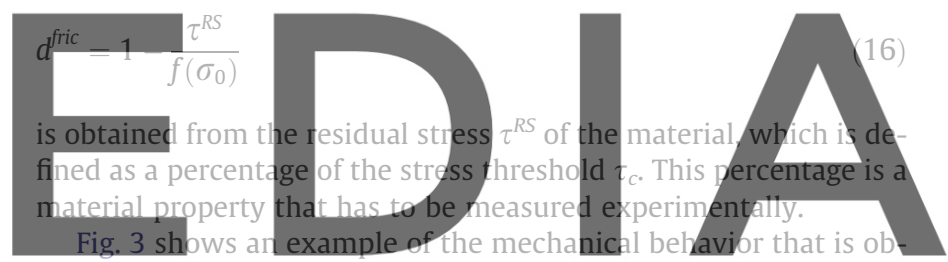

tained, in a unidirectional case, when a material is simulated with the frictional damage formulation just presented. This figure shows

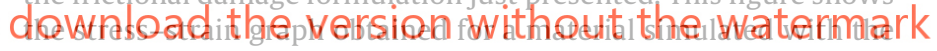

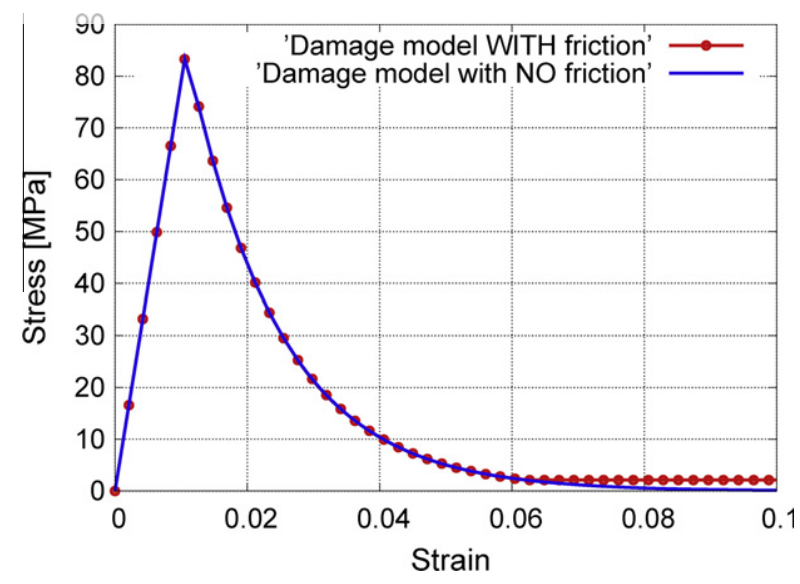

Fig. 3. Comparison of damage law with and without friction parameter.

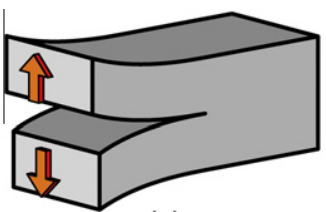

(a)

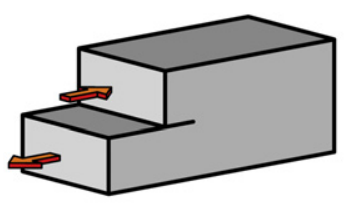

(b)

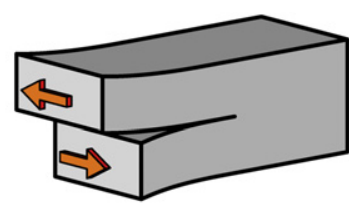

(c)

Fig. 2. Fracture modes: (a) mode I or opening mode, (b) mode II or shear mode and (c) mode III or tearing mode. 
friction damage model, and the graph obtained for the same material when it is simulated with the damage model without friction. The material considered corresponds to the matrix material that is used to validate the formulation, which is described in detail in Section 3.1. This material has a stress threshold of $85 \mathrm{MPa}$ and a frictional residual stress of $2.5 \%$ the threshold $(2.125 \mathrm{MPa})$.

It is important to notice that with the definition made of the friction parameter, the constitutive damage equation continues being isotropic; hence all stress directions have the same level of damage. This implies that in mode II fractures, like the one depicted in Fig. 2b, the formulation considers the friction effect but also a contact effect, as the remaining transverse stiffness on the fractured elements avoids the interpenetration of one side of the structure into the other one.

\section{Simulation of the ply drop-off test}

3.1. Test description

To validate the formulation presented in previous section, in the following are compared the results of an experimental test with the results obtained from a numerical simulation of this same test. The validation is made with the ply drop-off test. This test consists in applying a tensile force to a laminate with a variation of its thickness at its mid-span. A section with a change of thickness gives an eccentricity to the load applied, which generates a bending moment in the section containing the thickness variation.

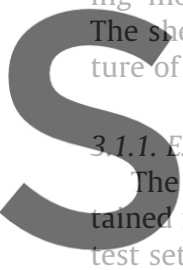
bear stress induced by this bend the specimen due to a delamin xperimental test experimental results of the ply drop-off test have been obfrom Airbus Spain, in the frame of the Femcom project. The represented in Fig. 4. The laminate used is made with the prepeg

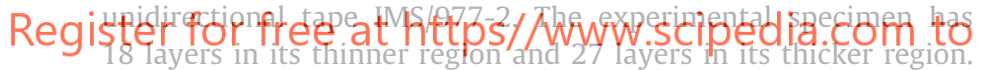
The thickness variation takes place in a length of $3.344 \mathrm{~mm}$. The stacking sequence of the laminate is depicted in Fig. 5, and the

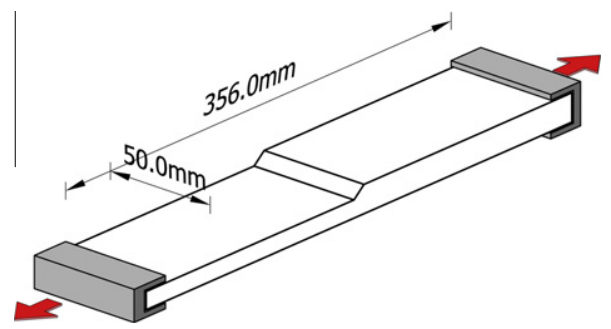

Fig. 4. Ply drop-off test specimen and load applied.
Table 3

Mechanical properties of the UD prepeg IMS/977.2.

\begin{tabular}{llllll}
\hline$E_{1}(\mathrm{GPa})$ & $E_{2}(\mathrm{GPa})$ & $E_{3}(\mathrm{GPa})$ & $G_{12}(\mathrm{GPa})$ & $G_{13}(\mathrm{GPa})$ & $G_{23}(\mathrm{GPa})$ \\
\hline 175.0 & 7.9 & 7.9 & 4.3 & 4.3 & 4.3 \\
& & & & $v$ & $t(\mathrm{~mm})$ \\
$G_{\text {Ic }}\left(\mathrm{J} / \mathrm{m}^{2}\right)$ & $G_{\text {IIc }}\left(\mathrm{J} / \mathrm{m}^{2}\right)$ & $G_{\text {III }}\left(\mathrm{J} / \mathrm{m}^{2}\right)$ & & 0.30 & 0.26 \\
555.0 & 738.0 & 738.0 & & & \\
\hline
\end{tabular}

material properties of the UD prepeg provided by the manufacturer are described in Table 3 . The specimen is loaded with a tensile force, which is applied with a controlled displacement.

\subsubsection{Numerical simulation}

The numerical simulation of the ply drop-off test is made using three-dimensional solid elements. In order to reduce the computational cost of the simulation, the solid laminate formulation previously described is used, staking layers in groups of three. Fig. 6 shows the element discretization along the thickness and the composite materials defined to each element. This figure shows that only two different solid laminate materials have to be defined:

Material 1: Composed by two layers at $0^{\circ}$ and one layer at $-45^{\circ}$ Material 2: Composed by two layers at $+45^{\circ}$ and one layer at $+90^{\circ}$.

It is worth to notice that the stacking sequence of Material 1 is

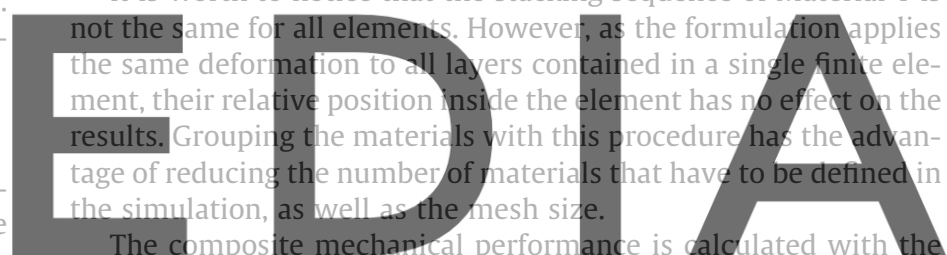
matrix-reinforced mixing theory (MR RoM). This theory requires knowing the mechanical properties of the composite components. dosmoloadntherverisjoravidithout thaukaternark ones of the prepeg, the matrix and fiber characteristics have been calculated from Table 3 using the following assumptions:

a. The contribution of fiber to the stiffness of the composite is only in fiber longitudinal direction.

b. Matrix is an isotropic material, with the same tensile and compression strength.

c. The fiber content in the composite is assumed to be $60 \%$

According to assumption (a), the composite properties in all directions, except in fiber longitudinal direction, are defined by matrix material. Therefore, matrix stiffness $E_{2}$ and $E_{3}$ must be coincident with composite $E_{2}$ and $E_{3}$, which are defined in Table 3. Because matrix is assumed to be isotropic (b), its stiffness in the longitudinal direction, $E_{1}$, is defined with the same value as $E_{2}$

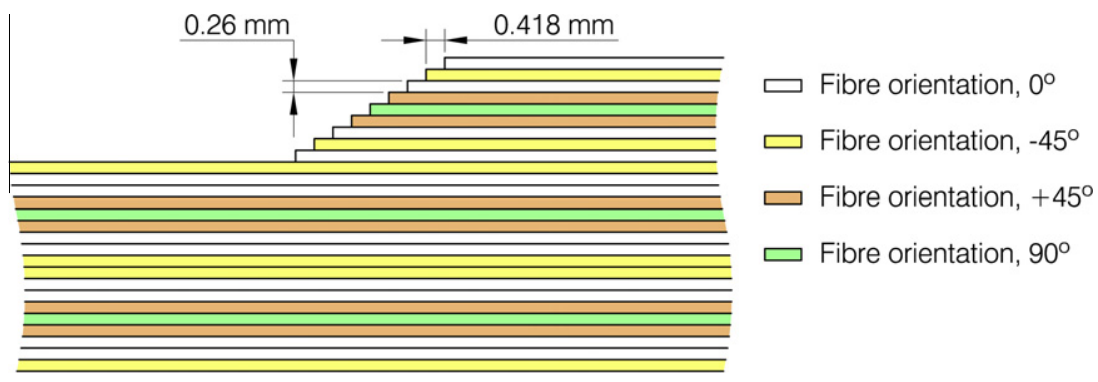

Fig. 5. Stacking sequence of the different layers of the laminate. 


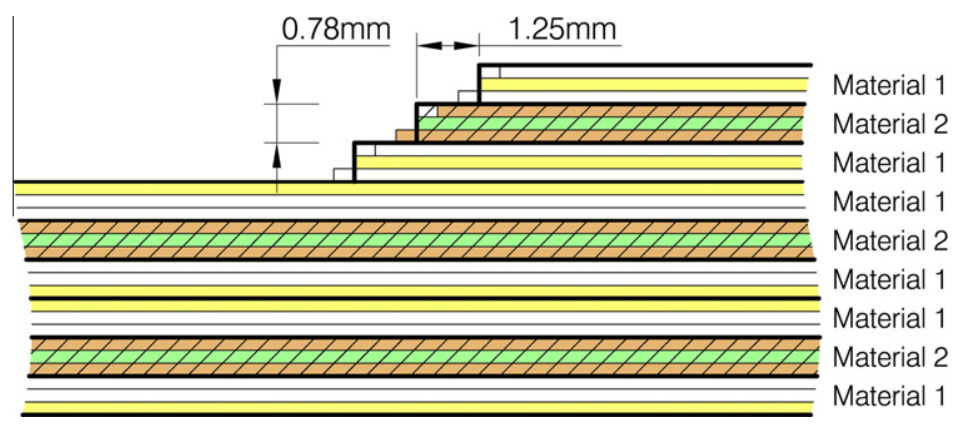

Fig. 6. Materials defined to conduct the numerical simulation of the ply drop-off test.

and $E_{3}$. Fiber longitudinal stiffness is obtained using the composite and matrix stiffness in longitudinal direction and the assumed fiber volumetric participation (c). Its value is obtained from the parallel compatibility equation of the MR RolM (Eq. (4)):

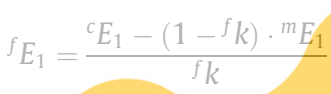

Table 4 contains a summary of the mechanical properties used to characterize each component material. The value of the matrix strength has been obtained from the characteristics provided by the manufacturer of the of $\mathrm{CYCOM}^{\circledR}$ 977-2 Toughened Epoxy Resin.

Fibers have been defined as a unidirectional elastic material. On

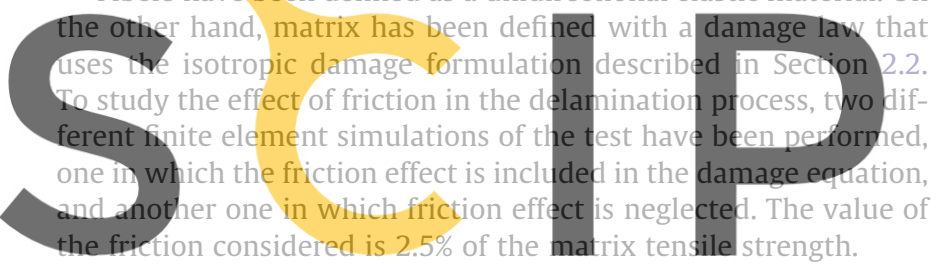

The mesh generated has 6120 linear hexahedrons and 7893 nodes. Based on the material division defined in.Fig. 6, the number

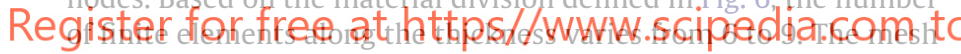
generated, as well as the materials defined, is depicted in Fig. 7. The boundary conditions applied to the model are the following: The longitudinal displacement of the left side of the specimen is fixed, and the right side is moved with a constant velocity during the

Table 4

Mechanical properties of the composite components.

\begin{tabular}{llll}
\hline Matrix properties & & Fiber properties \\
\hline Young modulus & $7.9 \mathrm{GPa}$ & Young modulus & $286.4 \mathrm{GPa}$ \\
Shear modulus & $4.3 \mathrm{GPa}$ & Poisson's ratio & 0.0 \\
Poisson's ratio & 0.3 & Volume content & $60.0 \%$ \\
Tensile strength & $85.0 \mathrm{MPa}$ & & \\
Fracture energy & $738.0 \mathrm{~J} / \mathrm{m}^{2}$ & & \\
Volume content & $40.0 \%$ & & \\
\hline
\end{tabular}

* Assumed value. whole simulation. The final displacement reached by the right side of the structure is $2.0 \mathrm{~mm}$.

\subsection{Results obtained}

\subsubsection{Comparison between the experimental and the numerical results}

The validity of the formulation proposed to simulate a delamination process is assessed comparing the force-displacement graph obtained from the experimental test and the numerical simulation. The force represented in the graph corresponds to the total tensile force applied to the sample. The displacement corresponds to the movement of the right end of the sample, result of the ap-

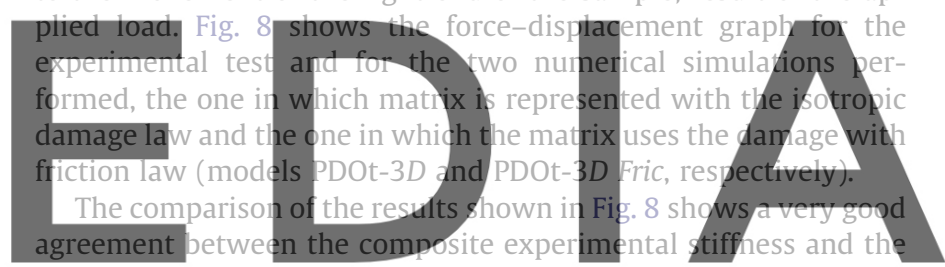

numerical stiffness obtained from the simulations. This figure also shows an oscillation in the force-displacement graph of the exper-

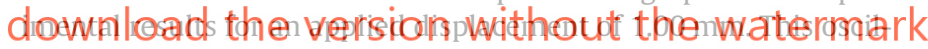
lation corresponds to delamination onset. The right side of the figure, in which the graph is magnified, shows that this oscillation appears aiso in the two numerical simulations performed, for an applied displacement of $1.15 \mathrm{~mm}$. In all results, numerical and experimental, the oscillation takes place for the same applied force of $85 \mathrm{kN}$. It will be further shown that in the numerical simulation, this force also corresponds to the load at which the delamination of the specimen begins. Therefore, both simulations are capable to predict the delamination onset with no error on the load, and with an error smaller than a $15 \%$ in the displacement. Fig. 8 shows that the agreement obtained in the sample stiffness, after the initiation of the delamination failure, is also very good.

Therefore, both numerical simulations match the initial stiffness of the laminate, the load at which delamination takes place, and the stiffness of the laminate once the delamination process has begun. This last stiffness is slightly larger in the simulation

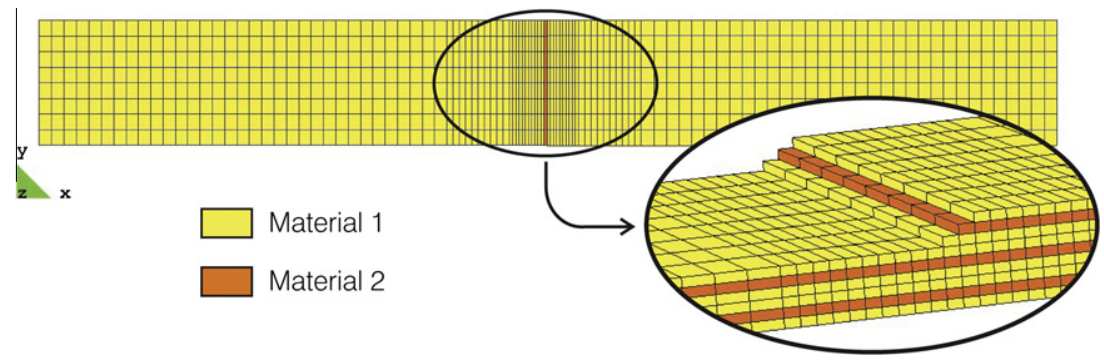

Fig. 7. Mesh defined to perform the numerical simulation. 

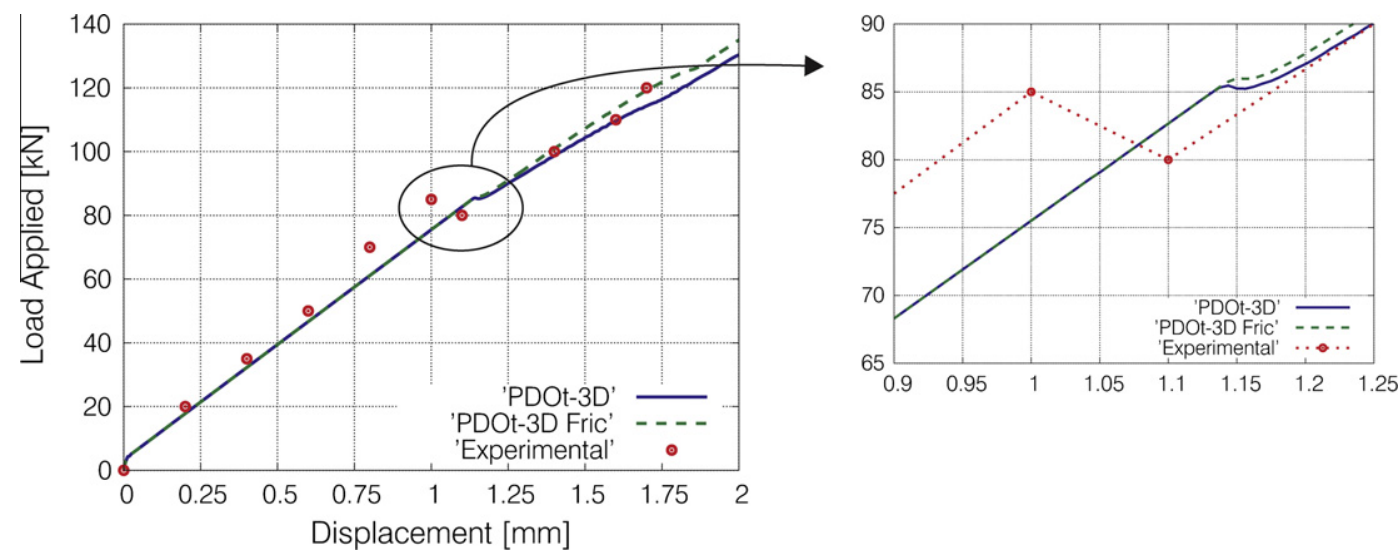

Fig. 8. Force-displacement graph of the experimental test and the numerical simulations.

in which the matrix material uses the friction damage formulation, as a result of the residual strength provided by friction. At the light of these results, it can be concluded that the formulation proposed is capable of simulating and predicting a delamination process in a laminated composite.

\subsubsection{Detailed study of the numerical results}

Once having assessed that the formulations proposed are valid to characterize delamination in composites, in the following are

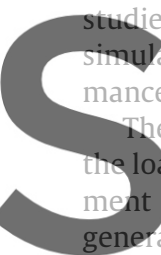

in detail the results obtained

ations, in order to have a bette

mance of these formulations.

The first thing that has to be validated is that the eccentricity of

e load, due to the specimen configuration, leads to a bending mo-

the numerical simulation; and that this bending moment ness variation. These two effects must be reproduced by the numerical simulation as these are the ones that will lead to the

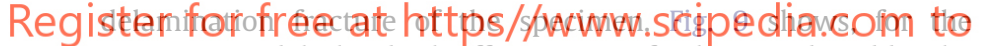
PDOt-3D model, that both effects are perfectly reproduced by the simulation. The existence of the bending moment is shown in Fig. 9a, where is represented the vertical deformation of the specimen for an applied displacement of $0.5 \mathrm{~mm}$. Fig. 9b shows the shear stresses in the specimen for the same applied displacement. In both figures the deformed structure has been magnified by 50 for a better visualization.

Once assessed that the effects that lead to delamination are properly represented by the numerical model, in the following is proved that the shear stresses shown in Fig. 9b are responsible of the delamination process, and that this delamination takes place for an applied longitudinal displacement of $1.15 \mathrm{~mm}$ (as shown in Fig. 8).

Delamination can be defined as the impossibility of transmitting shear stresses between two adjacent layers of the composite. In the matrix-reinforced mixing theory, the shear stresses of the composite are defined by the serial stresses of matrix material (Eq. (5)). When matrix is completely damaged it cannot develop

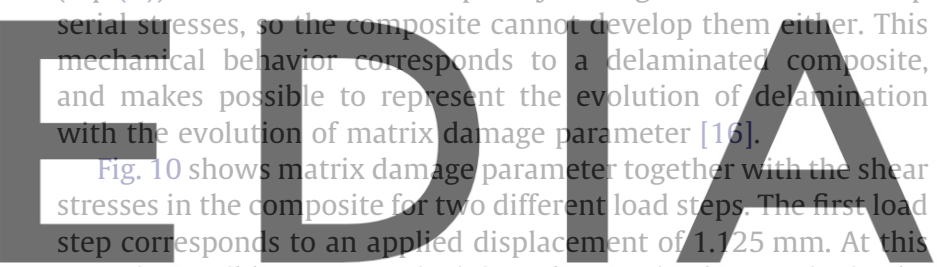
stage is possible to see an incipient damage in the matrix, in the first line of elements in which the composite thickness increases.

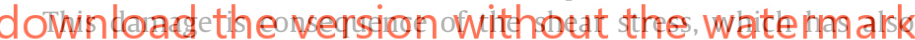
its maximum value in these elements. Next load step shows that matrix damage has propagated further inside the specimen or, in othei vords, it shovvs that delamination has begun and that it is progressing along the specimen. At this stage, shear stresses are zero in the damaged elements and they are maximum in the element adjacent to the last damaged one: the crack tip. These stresses are the responsible of delamination propagation. Therefore, Fig. 10 shows that the initiation of delamination in the specimen starts for a displacement between 1.125 and $1.150 \mathrm{~mm}$, coinciding

(a)
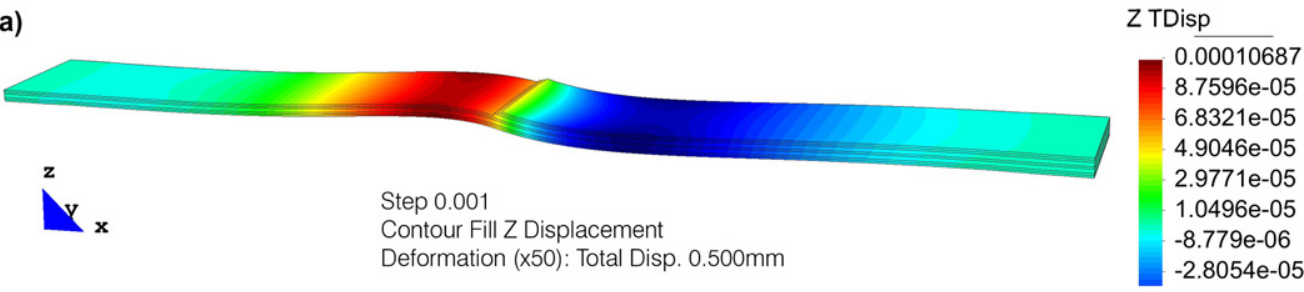

(b)

Sxz-N Stress

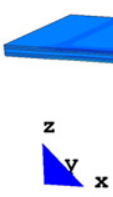

Contour Fill Z Displacement

Deformation (x50): Total Disp. 0.500mm

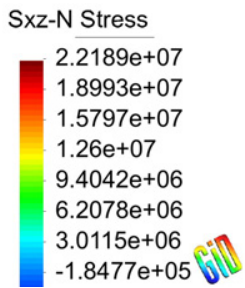

Fig. 9. Vertical displacement and shear stresses in the PDOt-3D model for an applied longitudinal displacement of $0.5 \mathrm{~mm}$ 
with the results shown by the force displacement graph depicted in Fig. 8.

Finally, Fig. 11 shows the evolution of delamination along the composite for the two models developed: PDOt-3D and PDOt-3D
Fric. Like in Fig. 10, delamination is represented by the matrix damage parameter. This figure proves that the formulation proposed not only is capable of characterizing delamination onset, but it is also capable of simulating the propagation of the delamination

(a) Displacement $=1.125 \mathrm{~mm}$
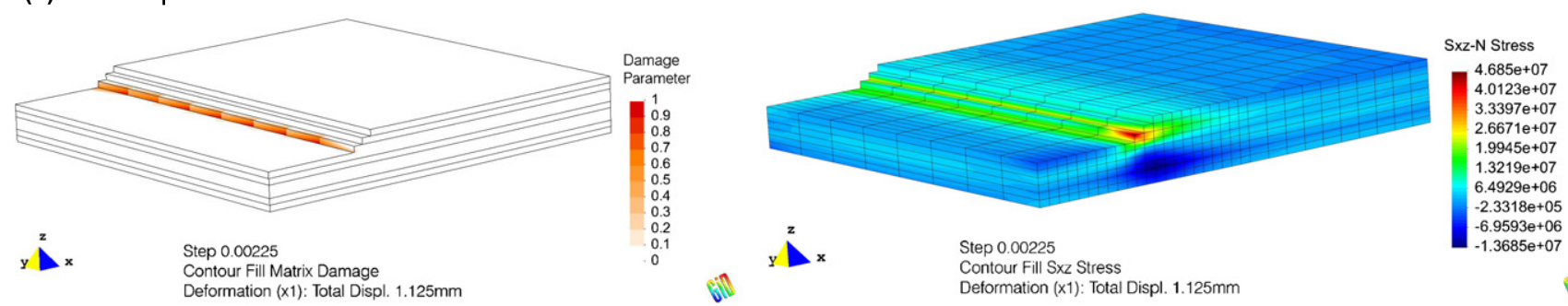
Deformation (x1): Total Displ. 1.125mm

Deformation (x1): Total Displ. $1.125 \mathrm{~mm}$

(b) Displacement $=1.150 \mathrm{~mm}$
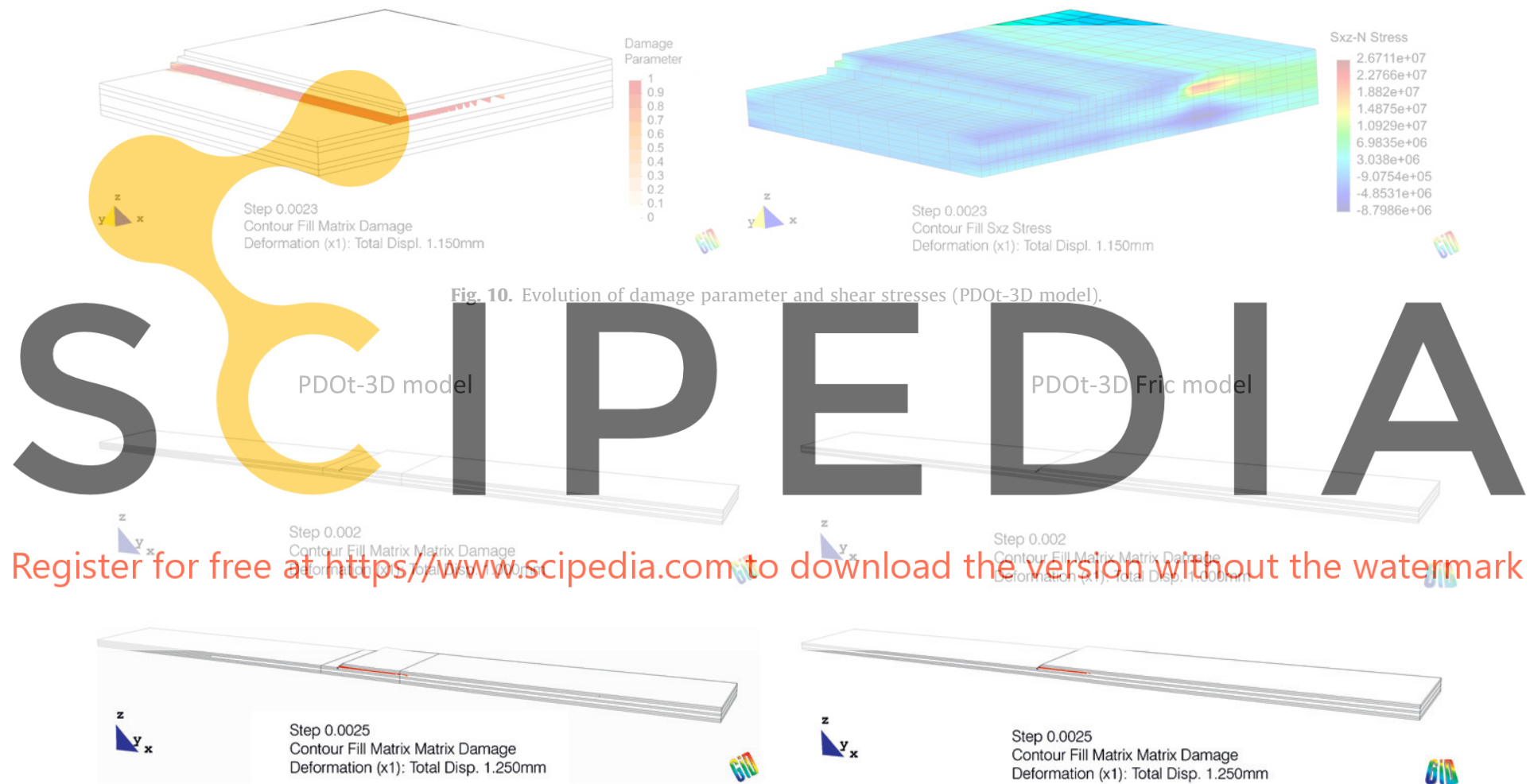

download thentwersiadrimonthout the watermark
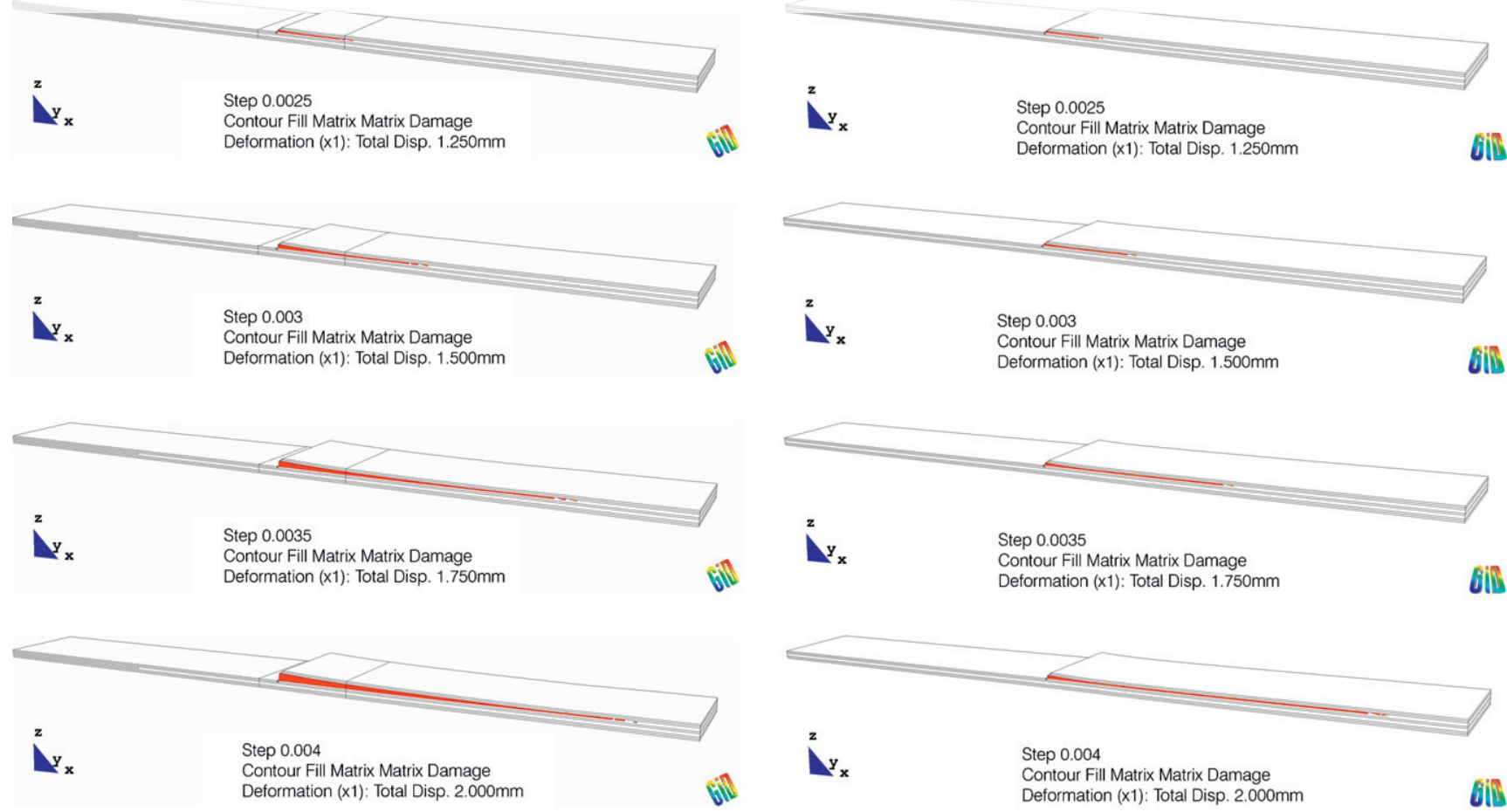

Fig. 11. Evolution of delamination in the PDOt-3D and the PDOt-3D Fric models. 
along the composite. Fig. 11 is also useful to compare the effect of friction in the damage formulation. While Fig. 8 shows that friction increases the stiffness of the laminate once delamination has started, this figure shows that delamination propagates slower when friction is considered, as matrix damage propagates slower in the PDOt-3D Fric model.

This effect is better shown in Fig. 12, in which is represented the delaminated length against the displacement applied to the right side of the specimen. This figure shows that although the final delaminated length is nearly the same $(166.25 \mathrm{~mm}$ when friction is considered, and $173.75 \mathrm{~mm}$ when friction is not considered), the velocity at which delamination propagates is considerably slower when friction is added to the damage formulation. The final delaminated length is nearly the same because, despite friction, the specimen cannot assume the total deformation applied to it. Therefore, during the last load steps of the simulation, the PDOt-3D Fric model shows an exponential increment of the delamination length.

\section{Conclusions}

This paper has presented several formulations developed to optimize the computational performance of an explicit finite element code. The resulting code is capable of predicting not only the elastic mechanical performance of composites but also their material non-linear behavior, including complex fracture processes such as delamination.
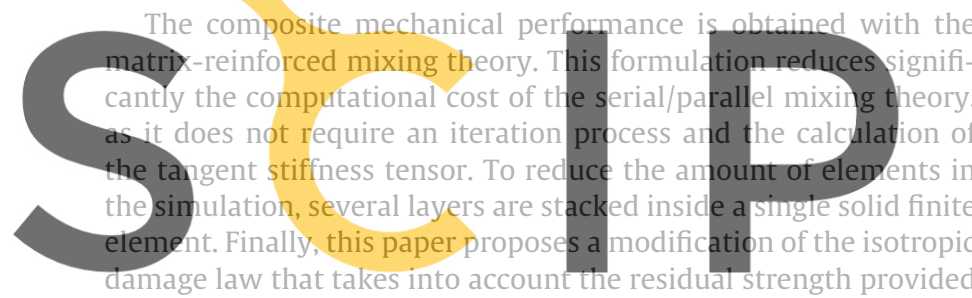

by friction in type II fracture modes

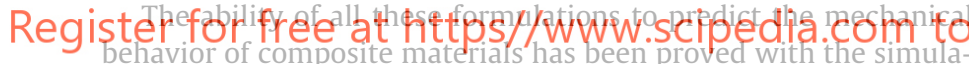

tion of the ply drop-off test. This test consists in applying a tensile force to a laminate with a thickness variation in its mid-span. The sample delaminates because of the efforts generated by the eccentricity in the load, result of the thickness variation. The comparison of the numerical results with experimental values of the test has shown that, not only the developed formulations are capable to successfully predict the elastic performance of the laminate, but they also provide an excellent prediction of the delamination failure: its onset and its propagation along the specimen. Friction,

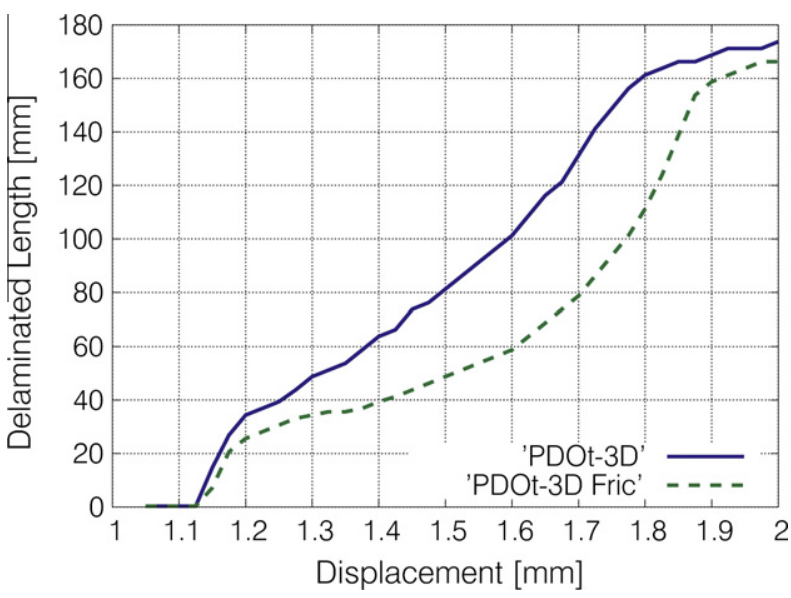

Fig. 12. Evolution of delamination length in the PDOt-3D and PDOt-3D Fric models which is accounted in the damage formulation, plays a role after delamination initiation akin to an increase in fracture toughness.

It is important to remark that the delamination failure has been simulated without having to pre-define the region where it is supposed to take place, and without having to add special elements to characterize it either. All elements in the simulation have the same material model and properties, and it is the formulation itself what predicts the delamination onset and its propagation along the specimen. Therefore, the proposed procedure and formulations not only are capable of optimizing the computational performance of an explicit finite element code, but they are also capable of successfully simulating complex failure modes such as delamination. This capability makes the numerical tool developed particularly well suited to perform large scale simulations of composite structures, such the ones used in the nautical and aeronautical fields, in which the computational cost of adding special elements to capture delamination problems may make the simulation computationally unaffordable with nowadays calculation capabilities

\section{Acknowledgements}

Most of the developments included in this work are consequence of several research Projects. The institutions and companies responsible of these Projects are gratefully acknowledged. These are the Spanish Government, through the Ministerio de Ciencia y Tecnología (RECOMP Project, ref. BIA2005-06952 and DECOMAR Project, ref. MAT2003-08700-C03-02), and the Ministe-

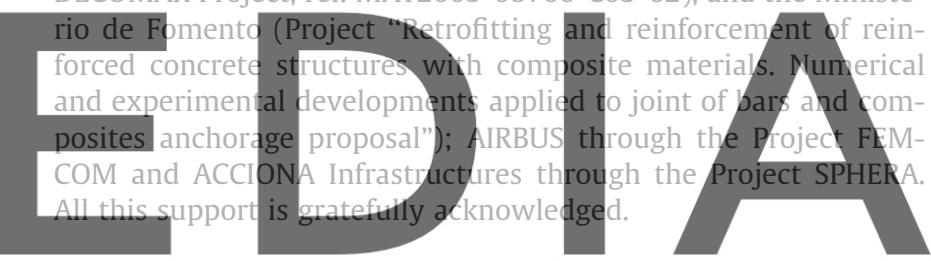

References

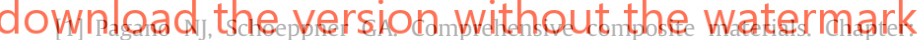
delamination of polymer matrix composites: problems and assessment. London, UK: Elsevier: 2000, 433-528.

2] Bolotin VV. Delamination in composite structures: its origin, buckling, growth and stability. Compos Part B: Eng 1996:27(2):129-45.

[3] Blanco N. Variable mixed-mode delamination in composite laminates under fatigue conditions: testing \& analysis. PhD thesis, Departament d'Enginyeria Mecànica I de la Construcció Industrial - Univeristat de Girona. Advisor: J. Costa; 2004.

[4] Turon A. Simulation of delamination in composites under quasi-static and fatigue loading using cohesive zone models. PhD thesis, Departament d'Enginyeria Mecànica I de la Construcció Industrial - Univeristat de Girona. Advisors: P.P. Camanho and J. Costa; 2006.

[5] Jansson NE, Larsson R. A damage model for simulation of mixed-mode delamination growth. Compos Struct 2001;53(4):409-17.

[6] Camanho PP, Dávila CG. Mixed-mode decohesion finite elements for the simulation of delamination in composite materials. NASA technical report TM2002-211737; 2002.

[7] Turon A, Camanho PP, Costa J, Dávila CG. A damage model for the simulation of delamination in advanced composites under variable-mode loading. Mech Mater 2006;38(11):1072-89.

[8] Balzani C, Wagner W. An interface element for the simulation of delamination in unidirectional fiber-reinforced composite laminates. Eng Fract Mech 2008;75(9):2597-615.

[9] Borg R, Nilsson L, Simonsson K. Modeling of delamination using a discretized cohesive zone and damage formulation. Compos Sci Technol 2002;62(1011):1299-314

[10] Borg R, Nilsson L, Simonsson K. Simulating DCB, ENF, and MMB experiments using shell elements and cohesive zone model. Compos Sci Technol 2004:64(2):269-78.

[11] Pinho ST, Iannucci L, Robinson P. Formulation and implementation of decohesion elements in an explicit finite element code. Compos Part A: Appl Sci Manufact 2006;37(5):778-89.

[12] Greco F, Lonetti P. Mixed mode dynamic delamination in fiber reinforced composites. Composites: Part B 2009;40:379-92.

[13] Barbero E, Sgambitterra G, Adumitroaiea A, Martinez X. A discrete constitutive model for transverse and shear damage of symmetric laminates with arbitrary stacking sequence. Compos Struct; 2010. doi:10.1016/j.compstruct. 2010.06.011. 
[14] Krueger R. Virtual crack closure technique: history, approach and applications. Appl Mech Rev 2004;57(2):109-43.

[15] Rastellni F, Oller S, Salomon O, Oñate E. Composite materials non-linear modeling for long fiber reinforced laminates: continuum basis, computational aspects and validations. Comput Struct 2008;86(9):879-96.

[16] Martinez X, Oller S, Barbero E. Mechanical response of composites. Chapter: study of delamination in composites by using the serial/parallel mixing theory and a damage formulation. Springer, ECCOMAS series edition; 2008

[17] Barbero EJ. Introduction to composite materials design. Philadelphia, USA: Taylor \& Francis; 1999.

[18] Jones RM. Mechanics of composite materials. 2nd ed. Taylor \& Francis: Philadelphia, USA; 1999.

[19] Martinez X, Oller S, Rastellini F, Barbat HA. A numerical procedure simulating RC structures reinforced with FRP using the serial/parallel mixing theory. Comput Struct 2008;86(15-16):1604-18.

[20] Martinez X, Oller S. Numerical simulation of matrix-reinforced composite materials subjected to compression loads. Arch Comput Methods Eng 2009;16(4):357-97.
[21] Dvorak GJ, Bahei-el-Din YA. Plasticity analysis of fibrous composites. J Appl Mech 1982;49:327-35.

[22] Oliver J, Cervera M, Oller S, Lubliner J. Isotropic damage models and smeared crack analysis of concrete. In: Second international conference on compute aided analysis and design of concrete structures. Zell am See, Austria; April 1990. p. $945-58$

[23] Oller S. Fractura mecánica. Un enfoque global. CIMNE, Barcelona, Spain; 2001

24] Lubliner J, Oliver J, Oller S, Oñate E. A plastic-damage model for concrete. Int Solids Struct 1989;25(3):299-326.

[25] Stringfellow RG, Freund LB. The effect of interfacial friction on the buckledriven spontaneous delamination of a compressed thin film. Int J Solids Struct 1993;30(10):1379-95.

[26] Dieter GE. Mechanical metallurgy. London, UK: McGraw-Hill Book Company: 1988.

[27] Davidson BD, Sun X. Effects of friction, geometry and fixture compliance on the perceived toughness from the three- and four-point bend end-notched flexure tests. J Reinforced Plastics Compos 2005;24(15):1611-28. 